\title{
Perfil socioeconômico e epidemiológico dos pacientes submetidos à cirurgia de catarata em um hospital universitário
}

\author{
Socioeconomic and epidemiologic profile of patients \\ submitted to cataract surgery at a university hospital
}

Beatriz de Abreu Fiuza Gomes', Ana Luiza Biancardi ${ }^{2}$, Camila Fonseca Netto ${ }^{3}$, Fernanda Ferreira Pires Gaffree $^{4}$, Haroldo Vieira de Moraes Junior

\section{Resumo}

Objetivo: Determinar as características sócioeconômicas dos pacientes operados de catarata em hospital universitário, avaliar o acesso à unidade terciária e determinar o tempo de espera até o tratamento cirúrgico. Métodos: Estudo transversal, realizado através da revisão de prontuários de 148 pacientes operados de catarata escolhidos aleatoriamente durante o primeiro semestre de 2007. Resultados: Os pacientes apresentaram idade mediana de 70,5 anos, com maior prevalência de mulheres $(60,1 \%)$, da raça branca $(48,0 \%$ ), com baixo grau de instrução (ensino fundamental incompleto, $50,7 \%)$, acuidade visual igual ou pior a 20/200 $(57,4 \%)$ e diagnóstico realizado em consulta regular no hospital universitário (47,8\%). Apenas 9,4\% dos pacientes operados foram diagnosticados na campanha de catarata. O tempo médio entre o diagnóstico e a cirurgia foi 3,5 meses e relacionou-se principalmente à realização dos exames oftalmológicos e clínicos pré-operatórios. Conclusão: Os pacientes com baixa renda familiar são os principais usuários do Sistema Único de Saúde (SUS) e o acesso ao tratamento cirúrgico é demorado, resultando em visão subnormal incapacitante. Ressalta-se a importância da campanha de catarata no ingresso de pacientes carentes ao hospital de alta complexidade.

Descritores: Extração de catarata/epidemiologia; Acessos aos serviços de saúde; Hospitais universitários; Agendamento de consultas

\footnotetext{
${ }_{2}^{1}$ Residente de Oftalmologia da Universidade Federal do Rio de Janeiro - UFRJ- Rio de Janeiro (RJ), Brasil; ${ }^{2}$ Médica ofatlmologista; Mestre pela Universidade Federal do Rio de Janeiro - UFRJ- Rio de Janeiro (RJ), Brasil; ${ }^{3}$ Pós-graduanda de Oftalmologia da Universidade Federal do Rio de Janeiro - UFRJ- Rio de Janeiro (RJ), Brasil; ${ }^{4}$ Residente de Oftalmologia da Universidade Federal do Rio de Janeiro - UFRJ- Rio de Janeiro (RJ), Brasil;

${ }^{5}$ Livre-docente, Professor adjunto da Universidade Federal do Rio de Janeiro - UFRJ- Rio de Janeiro (RJ), Brasil;
}

Trabalho realizado na Universidade Federal do Rio de Janeiro - UFRJ- Rio de Janeiro (RJ), Brasil.

Recebido para publicação em: 21/6/2008 - Aceito para publicação em 20/10/2008 


\section{INTRODUÇÃO}

A catarata é um distúrbio ocular provocado pela opacificação do cristalino. Dois mecanismos principais parecem agir na sua patogênese: estresse foto-oxidativo (da radiação UVA e UVB) ${ }^{(1)} \mathrm{e}$ estresse oxidativo (drogas lícitas e ilícitas, diabetes, tabagismo, alcoolismo) ${ }^{(2)}$. O fator de risco isolado mais expressivo é a idade avançada, pelo maior tempo de exposição a todos esses fatores ${ }^{(3-4)}$. A catarata é a principal causa mundial de cegueira, sendo responsável por $50 \%$ dos casos de incapacidade visual ${ }^{(5)}$. Há um número estimado de 17 milhões de cegos por catarata no mundo. Estima-se que a prevalência de cegueira por catarata deva ser duplicada até o ano de 2010, em razão da crescente expectativa de vida resultando no aumento da população idosa no mundo ${ }^{(6.8)}$. Em 1998, no Brasil, foi estimada a existência de 600 mil pessoas cegas por catarata, que se encontravam em duas situações básicas: sem diagnóstico estabelecido; ou com diagnóstico, à espera da realização do procedimento cirúrgico. De acordo com dados estatísticos do Conselho Brasileiro de Oftalmologia (CBO), existem, no nosso país, cerca de 350 mil pacientes acima de 50 anos, que estão cegos devido à catarata, sendo que, em $95 \%$ destes casos, a cegueira pode ser eliminada. Isso faz da cirurgia da catarata um dos procedimentos cirúrgicos mais realizados ${ }^{(9)}$. A quantidade estimada de cirurgias aumentou de 1.864.000 em 1999 para 2.122.800 em 2000, um acréscimo de 13,8\%. Entretanto, esse número expressivo ainda é baixo para atender a demanda anual de novos casos, que se somada ao déficit já acumulado durante anos, permite considerar a catarata como um importante problema de saúde pública ${ }^{(5-6)}$.

O objetivo do presente estudo foi determinar as características sócioeconômicas dos pacientes operados de catarata em hospital universitário, avaliar o acesso à unidade terciária e determinar o tempo de espera até o tratamento cirúrgico.

\section{Métodos}

Estudo transversal. Foram revisados os prontuários de 148 pacientes operados de catarata no Hospital Universitário Clementino Fraga Filho (HUCFF) entre 9 de janeiro a 9 de julho de 2007, escolhidos aleatoriamente através do número de registro, representando $41,6 \%$ das facectomias realizadas no período citado. Para caracterizar a amostra foram selecionadas as seguintes variáveis: idade, sexo, raça/etnia, estado civil, grau de instrução, tipo de atividade profissional, renda famili- ar mensal, acuidade visual pré-operatória, classificação da catarata do olho operado, estado do olho contralateral em relação ao diagnóstico de catarata e técnica cirúrgica empregada. Foram registrados ainda dados a respeito da forma e/ou origem de referência do paciente, do serviço onde foi feito o diagnóstico, do tempo decorrido entre o encaminhamento e a consulta, e do tempo de espera para cirurgia. Em caso de tempo de espera para a cirurgia maior que um mês, os principais motivos foram investigados.

\section{Resultados}

A idade variou de 36 a 89 anos, com média de 69 $+/-11,39$ anos, sendo que $91,8 \%$ tinham mais de 50 anos. Houve maior prevalência do sexo feminino $(60,1 \%)$. A raça branca foi responsável por $48,0 \%$ dos casos, seguida pela parda $(37,2 \%)$. A maioria dos pacientes $(54,7 \%)$ era casada (Tabela 1).

Em relação ao grau de instrução, dos 148 pacientes, somente dois pacientes $(1,4 \%)$ apresentavam curso superior completo. A maioria (50,7\%) apresentava ensino fundamental incompleto, seguida de 30,4\% com ensino fundamental completo, de $10,1 \%$ com ensino médio completo e de $6,8 \%$ de analfabetos (Gráfico 1 ).

Quanto à ocupação, 35,1\% dedicavam-se a atividades do lar e 29,7\% eram aposentados (Tabela 1).

A respeito da renda familiar, só foi possível obter informações de 83 prontuários, uma vez que elas são colhidas rotineiramente, mas não de forma obrigatória, durante a pré-admissão feita pelo serviço social. Todos os pacientes apresentavam renda familiar menor que 3000 reais, assim distribuída: $30,1 \%$ abaixo de 500 reais, $27,7 \%$ entre 501 a 1000 reais e $42,2 \%$ entre 1001 e 3000 reais (Tabela 1).

Quanto à melhor acuidade visual corrigida préoperatória do olho com catarata, aferida em tabela de Snellen, 2,0\% dos pacientes apresentavam visão melhor ou igual a $20 / 30 ; 17,6 \%$ visão entre $20 / 40$ a $20 / 60 ; 36,5 \%$ entre $20 / 80$ e $20 / 200$ e $43,9 \%$ pior ou igual a $20 / 400$ (Gráfico 2).

Em relação à classificação da catarata, 23,1\% apresentavam algum grau de catarata corticonuclear associada à catarata subcapsular posterior: $39,5 \%$, somente catarata corticonuclear; $25,9 \%$ catarata subcapsular posterior; $10,9 \%$, catarata branca e $0,7 \%$, catarata subcapsular anterior.

Para análise do grau de opacidade cristaliniana, consideramos a classificação do grau mais avançado descrito no prontuário de forma subjetiva variando de 1 
Tabela 1

Dados demográficos

\begin{tabular}{|c|c|c|}
\hline Perfil & $\mathbf{N}$ & $\%$ \\
\hline \multicolumn{3}{|l|}{ Sexo: } \\
\hline Sexo feminino & 89 & 60,1 \\
\hline Sexo masculino & 59 & 39,9 \\
\hline Total: & 148 & \\
\hline \multicolumn{3}{|l|}{ Etnia/raça } \\
\hline Branca & 71 & 48,0 \\
\hline Parda & 55 & 37,2 \\
\hline Negra & 17 & 11,5 \\
\hline Amarela & 5 & 3,4 \\
\hline Total: & 148 & \\
\hline \multicolumn{3}{|l|}{ Estado civil: } \\
\hline Solteira & 33 & 22,3 \\
\hline Casada & 81 & 54,7 \\
\hline Viúva & 34 & 23,0 \\
\hline Total: & 148 & \\
\hline \multicolumn{3}{|l|}{ Ocupação: } \\
\hline Aposentado & 44 & 29,7 \\
\hline Pensionista & 4 & 2,7 \\
\hline Autônomos & 8 & 5,4 \\
\hline Atividades do comércio & 5 & 3,4 \\
\hline Atividades do lar & 52 & 35,1 \\
\hline Desempregado & 32 & 21,6 \\
\hline Outras & 3 & 2,0 \\
\hline Total: & 148 & \\
\hline \multicolumn{3}{|l|}{ Renda familiar: } \\
\hline Inferior a 500 & 25 & 30,1 \\
\hline Entre 501 a 1000 reais & 23 & 27,7 \\
\hline Entre 1001 a 3000 reais & 35 & 42,2 \\
\hline Maior que 3000 reais & 0 & \\
\hline Total: & 83 & \\
\hline \multicolumn{3}{|l|}{ Classificação da catarata } \\
\hline Corticonuclear (CN) & 58 & 39,5 \\
\hline Subcapsular posterior (SCP) & 38 & 25,9 \\
\hline Branca & 16 & 10,9 \\
\hline Subcapsular anterior & 1 & 0,7 \\
\hline $\mathrm{CN}+\mathrm{SCP}$ & 34 & 23,1 \\
\hline Total: & 147 & \\
\hline \multicolumn{3}{|l|}{ Grau de Opacidade } \\
\hline 1 & 13 & 8,8 \\
\hline 2 & 51 & 34,5 \\
\hline 3 & 48 & 32,4 \\
\hline 4 & 17 & 11,5 \\
\hline Branca & 19 & 12,8 \\
\hline Total: & 148 & \\
\hline
\end{tabular}

até 4. Por exemplo, caso o paciente apresentasse catarata cortical grau 1 , nuclear grau 1 e subcapsular posterior grau 3, consideramos como grau 3 o grau de sua opacidade cristaliniana. Assim, 66,9\% dos pacientes apresentavam catarata grau 2 ou grau 3 .

A técnica cirúrgica mais realizada foi a facoemulsificação $(60,8 \%)$, seguida pela extração extracapsular $(35,1 \%)$ e pela cirurgia de facoemulsificação combinada com trabeculectomia $(4,1 \%)$.

Dos 148 pacientes, $35,1 \%$ já haviam realizado cirurgia de catarata no olho contralateral, sendo que a maioria $(75,0 \%)$ havia sido operada no próprio HUCFF. Dos $64,9 \%$ cujo olho contralateral era fácico, $61,5 \%$ apresentavam catarata com indicação cirúrgica, 30,2\% sem indicação cirúrgica e 8,3\% não apresentavam catarata.

$\mathrm{Na}$ maioria dos pacientes $(47,8 \%)$ foi feito o diagnóstico de catarata na consulta realizada no HUCFF. Em 35,5\% o diagnóstico foi feito em outro serviço público, em 2,2\%, em consulta oftalmológica por meio de plano de saúde, em 5,1\%, em clínicas particulares e somente $9,4 \%$ em campanhas de catarata.

Verificamos em relação a forma e/ou origem de referência do paciente que $33,3 \%$ foram encaminhados do posto de saúde ou de outra unidade pública; 40,6\%, via pedido de parecer de outros setores do HUCFF ; $25,4 \%$, consulta através de pedido de amigos e conhecidos e $0,7 \%$ provenientes de clínicas particulares.

O tempo de espera entre encaminhamento e consulta foi em média de $2+/-3,6$ meses. Já o tempo médio de espera entre consulta e cirurgia foi de 3,5+/- 8,1 meses. Nos casos em que o tempo de espera entre consulta e cirurgia ultrapassou 1 mês, foram investigados os principais motivos relacionados a demora, sendo que em 11 casos não foi possível obter esta informação.

A demora em agendar o risco cirúrgico foi verificada em $35,6 \%$ dos prontuários sendo que em $16,3 \%$ foi agendar exames oftalmológicos pré-operatórios (ecobiometria, microscopia especular); em 16,3\% foi disponibilizar vagas para cirurgia e em $11,1 \%$ houve relatos de demora por motivos pessoais, como dúvidas a respeito da decisão de operar, por exemplo. Outros motivos foram encontrados em $20,7 \%$, como adiamento da cirurgia por questões administrativas do hospital, pacientes que não encontravam-se em condições satisfatórias do ponto de vista clínico para realização de cirurgia, entre outros.

\section{Discussão}

A catarata é a principal causa de cegueira no mundo ${ }^{(5)}$, apesar de ser passível de recuperação por intervenção cirúrgica relativamente simples e de baixo custo. Segundo dados do Ministério da Saúde, existiam em 1998, 600 mil pessoas apresentando cegueira devido à catarata e uma estimativa de aparecimento de 120 mil novos casos por ano. Até 1998, os procedimentos cirúrgicos de catarata eram realizados apenas como ro- 


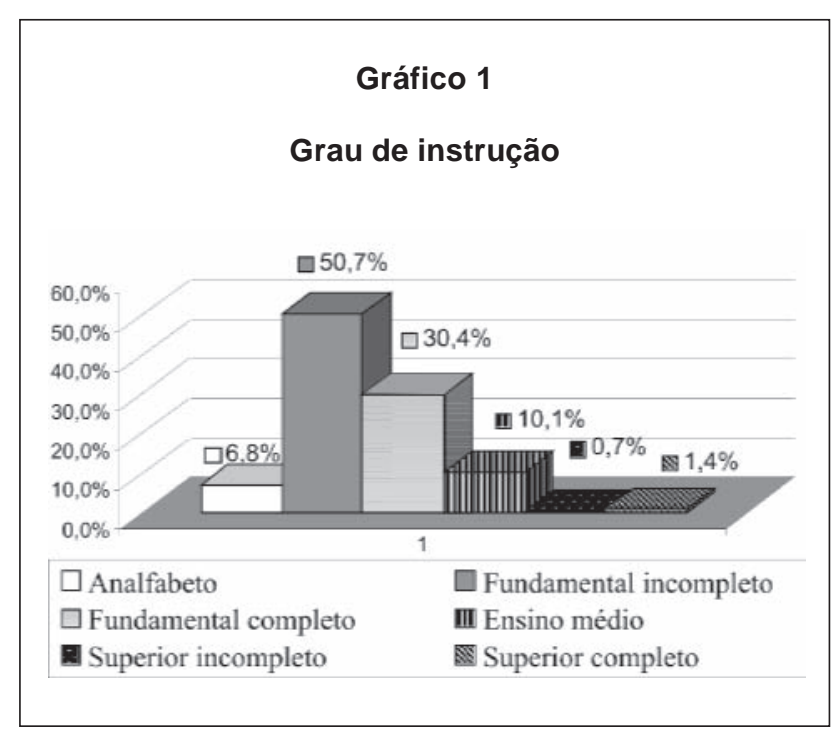

tina nas unidades de saúde pública. No caso específico da catarata, a espera podia ser de até sete anos ${ }^{(10)}$. As repercussões sociais, psicológicas e econômicas dessa situação são imensas. A campanha de redução de demandas reprimidas, que se iniciou no ano de 1999, foi introduzida com os objetivos de redução das filas de espera, de aumento da oferta de procedimentos eletivos e de aumento e melhora no acesso dos usuários do Sistema Único de Saúde (SUS) à assistência médica ${ }^{(11)}$.

Em 1999 foram realizadas 295.700 cirurgias de catarata no Brasil. Dessas, 153 mil (51,7\%) foram feitas pela campanha e as demais $(48,3 \%)$ na rotina ${ }^{(12)}$. Porém, em fevereiro de 2006 o Ministério da Saúde pôs fim ao programa de mutirões, única resposta efetiva até então para tratamento em massa da população carente, criando um déficit de $80 \%$ no número de cirurgias ${ }^{(13)}$. Esse dado é corroborado pelo nosso estudo, visto que dos pacientes operados no serviço de oftalmologia do HUCFFUFRJ no período de 09/01/2007 a 09/07/2007 a maioria $(66,0 \%)$ eram oriundos do próprio hospital e somente $29,5 \%$ tinham sido atendidos em consulta de $1^{\mathrm{a}}$ vez em catarata, isto é, vagas destinadas a pacientes encaminhados de outras unidades de saúde com a suspeita diagnóstica da catarata.

Em relação ao diagnóstico da catarata, $47,8 \%$ dos pacientes tiveram o seu diagnóstico firmado apenas na consulta no HUCFF, enquanto $34,1 \%$ o tiveram no serviço público básico e 9,4\% na avaliação da $1^{\mathrm{a}}$ vez em catarata. Apenas 5,1\% pacientes tiveram avaliação e diagnóstico prévio de catarata em serviço privado. Esses dados coincidem com estimativas feitas na cidade de São Paulo em que 3\% da população brasileira tem acesso à medicina privada e $80 \%$ apenas à assistência

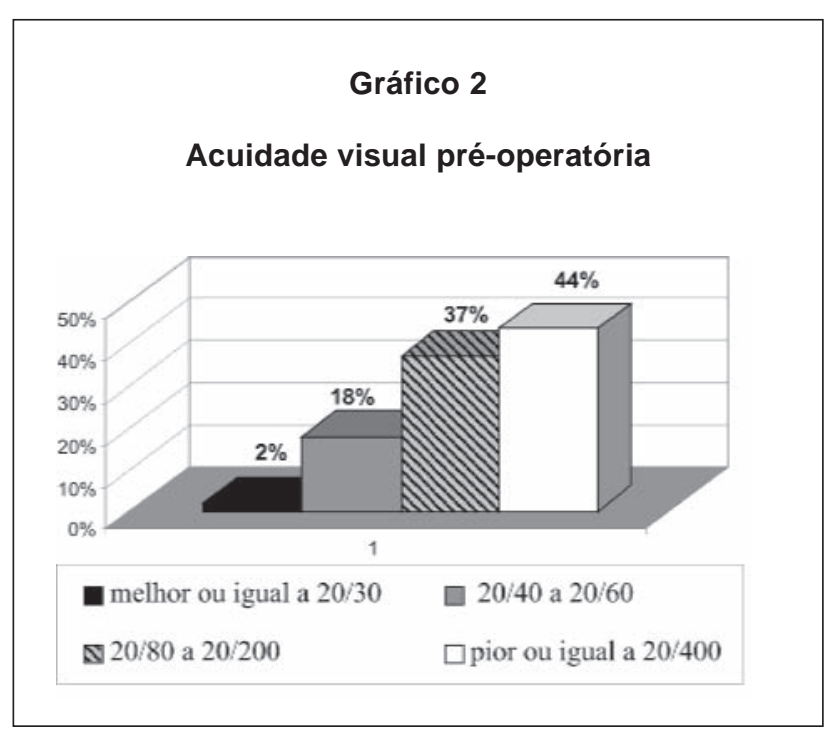

médica pública ${ }^{(14)}$. Ao avaliarmos o local de origem do encaminhamento do paciente ao nosso serviço, verificamos um percentual maior de pacientes encaminhados via parecer interno de dentro do próprio hospital (40,6\%), o que demonstra uma demanda interna muito grande nos hospitais universitários.

Em relação ao perfil socioeconômico dos pacientes do estudo, foi visto que a média da idade foi de 69 anos, que $60,1 \%$ eram do sexo feminino e $48,0 \%$ da raça branca. Esses dados são semelhantes aos observados em outros estudos ${ }^{(10,15-17)}$. Segundo censo demográfico de 2000 existe mais mulheres idosas do que homens idosos no Brasil ${ }^{(18)}$, o que também já foi evidenciado em estudos epidemiológicos, realizados em São Paulo e Pernambuco, com resultados de maior número de portadores de catarata no sexo feminino ${ }^{(10,17)}$.

Os dados relativos à escolaridade e ocupação demonstraram que a maioria dos pacientes possuía escolaridade baixa e eram aposentados ou do lar, além de possuírem baixa renda familiar. Isso demonstra o grau elevado de carência da população assistida pelos hospitais públicos, sendo estes economicamente inativos e com grande dificuldade de acesso a saúde. O mesmo resultado foi encontrado em estudos epidemiológicos feitos no estado de São Paulo e Pernambuco, mostrando que este problema possivelmente é vivenciado em todo o Brasil ${ }^{(10,17)}$.

Observamos também que $57,4 \%$ dos pacientes chegaram ao nosso serviço com acuidade visual pior ou igual a 20/200 e 44\% com acuidade visual pior ou igual que 20/400, demonstrando mais uma vez a provável demora no atendimento desses pacientes e todas as prováveis dificuldades que essa baixa visual avançada 
acarreta na vida dessas pessoas.

O tempo médio de espera entre a consulta oftalmológica e o dia da cirurgia foi 3,5 meses. Os motivos da espera foram, na maioria das vezes, devido à demora na realização de exames pré-operatórios (risco cirúrgico e exames oftalmológicos), o que correspondeu a 51,9\%. Outro motivo de demora encontrado neste estudo foi relacionado a problemas pessoais $(11,1 \%)$, já que muitos pacientes possuem medos e conceitos errados sobre a cirurgia de catarata. Estudos anteriores mostraram que muitos pacientes com diagnóstico prévio de catarata não procuraram atendimento para realização de cirurgia por receio de complicações e cegueira ${ }^{(19-20)}$.

Sobre o tipo de cirurgia mais realizada no nosso serviço, $60,8 \%$ foram facectomias pela técnica de facoemulsificação e $35,1 \%$ pela técnica de extração extracapsular. Isso provavelmente se deu pelas vantagens da primeira técnica, já que a técnica de facoemulsificação permite o uso de anestesia tópica, reduz o número de retornos e o tempo de duração do período pós-operatório. Além disso, a utilização da facoemulsificação otimiza o centro cirúrgico por tornar a cirurgia mais rápida e embora os custos totais da cirurgia de catarata mediante a técnica de facoemulsificação sejam maiores que da cirurgia por extração extracapsular ${ }^{(21)}$, já foi demonstado em estudo de análise de custo realizado em um hospital público de São Paulo, que a remuneração efetuada pelo SUS para a cirurgia por facoemulsificação pode apresentar vantagens econômicas em relação a cirurgia por extração extracapsular ${ }^{(21)}$.

\section{ConClusão}

Apesar do empenho no combate à catarata, a população desfavorecida do ponto de vista socioeconômico ainda não tem acesso rápido ao tratamento cirúrgico, o que resulta em visão subnormal incapacitante com repercussões sociais, psicológicas e econômicas. Ressaltase a importância da campanha de catarata no ingresso dos pacientes ao hospital de alta complexidade.

\section{Abstract}

Purpose: To determine the socio-economic characteristics of the patients who were submitted to cataract surgery in a university hospital, to evaluate the accessibility to the tertiary health service and to determine the waiting time for cataract surgery. Methods: A cross-sectional study of medical charts from 148 random patients who underwent cataract surgery during the first semester of 2007. Results: The patients in this study had median of age of 70.5 years, and the major prevalence was of female $(60.1 \%)$, caucasian $(48.0 \%)$, with low educational level (incomplete fundamental study, 50.7\%), best corrected visual acuity 20/200 or worst; the diagnosis of cataract was done at the University Hospital in a routine appointment $(47.8 \%)$. Only $9.4 \%$ of patients submitted to the surgery had his diagnosis done in a cataract campaign. The average time between the diagnosis and the surgery was 3.5 months and the delay was mainly due to pre operative exams. Conclusion: The majority of patients in the Brazilian Public Health System have low family income. The system takes a long time to access surgical procedures that need to be addressed in a proper time manner. The result of the long wait is subnormal vision. It is important to highlight the cataract campaign in order to enroll patients with low income in a hospital of high complexity.

Keywords: Cataract extraction/epidemiology; Health services accessibility; Hospitals, university; Appointments and schedules

\section{REFERÊNCIAS}

1. Bhuyan KC, Bhuyan DK. Molecular mechanism of cataractogenesis: III. Toxic metabolites of oxygen as initiators of lipid peroxidation and cataract. Curr Eye Res. 1984;3(1):67-81.

2. Leske MC, Chylack LT Jr, Wu SY. The Lens Opacities CaseControl Study. Risk factors for cataract. Arch Ophthalmol. 1991;109(2):244-51.

3. Tasman W, Jaeger EA. Duane's Ophthalmology: 2000 [CDROM]. 23th ed. Philadelphia: Lippincott Williams \& Wilkins; 2000. Section 3: Diseases of the lens.

4. Richter-Mueksch S, Zehetmayer M, Radner W, Kaider A, Velikay-Parel M. Influence of sex, visual acuity, and systemic disease on delayed presentation for cataract surgery in Austria. J Cataract Refract Surg. 2001;27(12):1999-2005.

5. World Health Organization. Department of Chronic Diseases and Health Promotion (CHP). Blindness: Vision 2020. Global Initiative for the Elimination of Avoidable Blindness [Internet]. Geneva: WHO; [updated 2000 Fev; cited 2003 Fev 13]; [about 8 screens].Available from: http://www.who.int/ mediacentre/factsheets/fs213/em/

6. Kara-José N, Temporini ER. Catarata e cegueira: epidemiologia e prevenção. In: Arieta CEL. Cristalino e catarata. Rio de Janeiro: Cultura Médica; 2002. p.50-6

7. Sommer A. Public health aspects of ophthalmic disease: introduction. Annu Rev Public Health. 1996;17:115-9.

8. Javitt JC, Wang F, West SK. Blindness due to cataract: epidemiology and prevention. Annu Rev Public Health. 1996;17:159-77.

9. Management of functional impairment due to cataract in adults. Cataract Management Guideline Panel. Ophthalmology. 1993;100(8 Suppl):1S-350S. 
10. Silva LMP, Muccioli C, Belfort Júnior R. Perfil socioeconômico e satisfação dos pacientes atendidos no mutirão de catarata do Intituto da Visão - UNIFESP. Arq Bras Oftalmol. 2004;67(5):737-44.

11. Brasil. Ministério da Saúde. Redução de demandas reprimidas: mutirões [Internet]. Brasília: Ministério da Saúde; [citado 2002 Set 4]; [cerca de 3 telas]. Disponível em: http:// saude.gov.br/sas/relatorio/6.4\%20demandas.htm

12. Brasil. Ministério da Saúde. Relatório. Jornal Oftalmológico Jota Zero [Internet]2002. [citado 2008 Set 1]; edição 86. Disponível em: http://www.cbo.com.br/jotazero/ed86/ relatorio.htm

13. Demagogia populista engana população carente. Jornal Jota Zero. 2006; maio/junho. p.9 .

14. Kara-José N, Alves MR. Catarata: um problema de saúde pública. In: Kara-José N, organizador. Prevenção da cegueira por catarata. Campinas: UNICAMP; 1996. p.11-8.

15. Zacharias LC, Graziano RM, Oliveira BFT, Hatanaka M, Cresta FB, Kara-José N. A campanha de catarata atrai pacientes da clínica privada? Arq Bras Oftalmol. 2002;65(5):557-61.

16. Lima DMG, Ventura LO, Brandt CT. Barreiras para o acesso ao tratamento da catarata senil na Fundação Altino Ventura. Arq Bras Oftalmol. 2005;68(3):357-62.
17. Ventura LO, Brandt CT. Projeto Mutirão de Catarata em centro de referência oftalmológico, em Pernambuco: perfil, grau de satisfação e benefício visual do usuário. Arq Bras Oftalmol. 2004;67(2):231-5.

18. Instituto Brasileiro de Geografia e Estatística. Censo demográfico 2000. Resultados preliminares da amostra. População [citado 2002 nov 11]. Disponível em: http:// www.ibge.gov.br .

19. Oliveira Rde S, Temporini ER, Kara José N, Carricondo PC, Kara José AC. Perceptions of patients about cataract. Clinics. 2005;60(6):455-60.

20. Temporini ER, Kara N Jr, Jose NK, Holzchuh N. Popular beliefs regarding the treatment of senile cataract. Rev Saude Publica. 2002;36(3):343-9.

21. Kara-José Junior N, Avakian A, Lower LM, Rocha AM, Cursino M, Alves MR. Facoemulsificação versus extração extracapsular manual do cristalino: análise de custos . Arq Bras Oftalmol.2004;67(3):481-9. 